\title{
A GENERAL WEDDERBURN THEOREM
}

\author{
BY CARL FAITH
}

Communicated by S. Smale, May 27, 1966

Let $R$ be a ring, $E$ a left $R$-module, and set

$$
R^{\prime}(E)=R^{\prime}=\operatorname{End}_{R} E, \quad R^{\prime \prime}(E)=R^{\prime \prime}=\operatorname{End}_{R^{\prime}} E .
$$

We say that $E$ is balanced in case the natural homomorphism $\lambda: R \rightarrow R^{\prime \prime}$ under which $x \mapsto \lambda_{x}$ where $\lambda_{x}(v)=x v, \forall v \in E$, is an isomorphism. The classical Wedderburn theorem gives a criterion for a module to be balanced. We give a very short proof of a theorem of Morita (in the terminology of [1]) which implies many such criteria.

A left $R$-module $E$ is said to be a generator (for left $R$-modules) if every $R$-module can be expressed as a homomorphic image of (possibly infinite) direct sum of copies of $E$.

ThEOREM 1. Let $E$ be a generator. Then $E$ is balanced.

Proof. We first prove that for any module $F, R \oplus F$ is balanced. Given $v \in R \oplus F$, there exists an element $\phi \in R^{\prime}(R \oplus F)$ such that $\phi(1)=v$ (we view $R$ and $F$ as embedded in $R \oplus F$ as $R \oplus 0$ and $0 \oplus F$ respectively). Let $p: R \oplus F \rightarrow R$ be the projection. Let $f \in R^{\prime \prime}(R \oplus F)$. Then $f(1)=f p(1)=p f(1)$. Hence $f(1) \in R$. It follows that

$$
f(v)=f \phi(1)=\phi f(1)=\phi(f(1) \cdot 1)=f(1) \phi(1)=f(1) v .
$$

This proves what we wanted.

Let $E$ be a generator. There exists a surjective homomorphism $E^{n} \rightarrow R$ for some integer $n \geqq 1$ (we can take $n$ finite because $R$ is generated by one element). Since $R$ is in fact free, we can write $E^{n}=R \oplus F$ for some module $F$. Hence $E^{n}$ is balanced. We conclude the proof with the following lemma.

LEMMA. If $E$ is any module and $E^{n}$ is balanced, then $E$ is balanced.

Proof. An element $\phi \in \operatorname{End}_{R}\left(E^{n}\right)$ can be represented by a matrix $\left(\phi_{i j}\right)$ with $\phi_{i j} \in \operatorname{End}_{R}(E)$, namely for $v \in E^{n}$ with components $v_{j} \in E$ we have

$$
\phi(v)=\left(\begin{array}{lll}
\phi_{11} & \cdots & \phi_{1 n} \\
\vdots & & \vdots \\
\phi_{n 1} & \cdots & \phi_{n n}
\end{array}\right)\left(\begin{array}{c}
v_{1} \\
\vdots \\
v_{n}
\end{array}\right) .
$$

Let $f \in R^{\prime \prime}(E)$. Then the matrix 


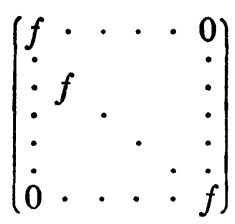

operating on $E^{n}$ commutes with every $\phi=\left(\phi_{i j}\right) \in R^{\prime}\left(E^{n}\right)$, and hence there exists an element $x \in R$ such that $(f)=\left(\lambda_{x}\right)$. This proves our lemma.

As applications of Theorem 1, we have the following special cases.

EXAMPLE 1. Let $R$ be a ring without two-sided ideals except 0 and $R$. Then any left ideal $L \neq 0$ is a generator, because $L R=R$ and hence there exists elements $a_{i} \in R(i=1, \cdots, n)$ such that $R=\sum_{1}^{n} L a_{i}$, whence a surjective homomorphism $L^{n} \rightarrow R$. In this way we recover Rieffel's theorem [6].

ExAMPLE 2. Let $R$ be a Dedekind ring, and $I$ any fractional ideal $\neq 0$. Then $I$ is a generator, again because $I I^{-1}=R$, so there exist elements $a_{i}$ in the quotient field of $R$ such that $R=\sum I a_{i}$.

We next prove a converse for Theorem 1 (Morita [5]).

Theorem 2. $A$ module $E$ is a generator if and only if $E$ is balanced as an $R$-module, and finitely generated projective as an $R^{\prime}$-module.

Proof. Let $E$ be a generator. We have already proved that $E$ is balanced, and we have a left $R$-module isomorphism $E^{n} \simeq R \oplus F$ for some module $F$. Applying $\operatorname{Hom}_{R}(\cdot, E)$ to $E^{n}$, we find the natural additive group isomorphisms

$$
\begin{aligned}
R^{\prime n} & =\operatorname{Hom}_{R}(E, E)^{n} \simeq \operatorname{Hom}_{R}\left(E^{n}, E\right) \\
& \simeq \operatorname{Hom}_{R}(R, E) \oplus \operatorname{Hom}_{R}(F, E) .
\end{aligned}
$$

Each one of the abelian groups appearing in (1) is in fact a left $R^{\prime}$ module, the operation of $R^{\prime}$ on each group on the left being defined as composition of mappings. (This is trivially verified.) Furthermore the isomorphisms in (1) are compatible with this operation. Therefore (1) is a statement of left $R^{\prime}$-isomorphism and direct sum. Furthermore $\operatorname{Hom}_{R}(R, E)$ is $R^{\prime}$-isomorphic to $E$ (under the map $f \mapsto f(1)$ ). Hence $E$ is $R^{\prime}$-finitely generated (being an $R^{\prime}$-homomorphic image of $R^{\prime n}$ ) and $R^{\prime}$-projective by (1). Conversely, if $R^{\prime n} \approx E \oplus F$ for some $R^{\prime}$-module $F$, and $R \simeq \operatorname{Hom}_{R^{\prime}}(E, E)$, then

$$
\operatorname{Hom}_{R^{\prime}}\left(R^{\prime n}, E\right) \simeq\left[\operatorname{Hom}_{R^{\prime}}\left(R^{\prime}, E\right)\right]^{n}=E^{n}
$$

yields an isomorphism

$$
E^{n} \simeq R \oplus G
$$


of left $R^{\prime}$-modules, where $G=\operatorname{Hom}_{R^{\prime}}(F, E)$. This proves that $E$ is a generator.

A simple ring is a ring (with identity) without two-sided nontrivial ideals.

THEOREM 3. If $R$ is a simple ring, and if $I$ is a left ideal $\neq 0$, then $I$ is finitely generated projective over $K=\operatorname{End}_{R} I$, and $R \simeq_{\text {nat }}$ End $_{K} I$. Furthermore, $K$ is a simple ring if and only if $I$ is a finitely generated projective right ideal, and in this case there is a category isomorphism ${ }_{R} \mathfrak{N} \simeq_{K} \mathfrak{T l}$.

Proof. The first sentence follows from Example 1 and Theorem 2. Also, if $I_{R}$ is finitely generated projective, then $K$ is known to be simple along with $R$. Conversely, $I$ is homomorphic to a left ideal $I^{\prime}$ of $K$ (under right multiplication by elements of $I$ ). If $K$ is simple, then ${ }_{K} I^{\prime}$, whence ${ }_{K} I$, is a generator. Since $R \simeq \operatorname{End}_{K} I, I_{R}$ is finitely generated projective by Theorem 2 . Since $I$ is then a progenerator, ${ }_{K} \mathfrak{T} \simeq_{R} \mathfrak{T C}$ follows (Morita [5]).

This theorem sharpens theorems of M. Rieffel [6], R. Hart [4], and Faith [3].

We conclude with another application of Theorem 1.

Example 3. A Dedekind domain $R$ is integrally closed. For if $S$ is a ring extension of $R$ contained in the quotient field of $R$, and if $S_{R}$ is finitely generated, then $S_{R}$ is a generator by Example 2, so $R$ $\simeq_{\text {nat }}$ End $_{R^{\prime}} S$. But obviously $R^{\prime}=\operatorname{End}_{R} S \simeq_{\text {nat }} S$, and End $S \simeq_{\text {nat }} S$. Thus $S=R$.

It is remarkable that Theorem 2 should imply both Theorem 3 and Example 3.

\section{REFERENCES}

1. H. Bass, On the Morita theorems, mimeographed notes, University of Oregon, 1962.

2. C. W. Curtis and I. Reiner, Representation theory of finite groups and associative algebras, Interscience, New York, 1962, p. 176, 405.

3. C. Faith, Noetherian simple rings, Bull. Amer. Math. Soc, 70 (1964), 730-731.

4. R. Hart, Simple rings with uniform right ideals, Proc. London Math. Soc. (to appear).

5. K. Morita, Duality for modules and its applications to the theory of rings with minimum condition, Science Reports, Tokyo Kyoiku Daigaku Sec. A (1958), 83-142.

6. M. A. Rieffel, $A$ general Welderburn theorem, Proc. Nat. Acad. Sci. USA 54 (1965), 1513.

University of California, Berkeley and Rutgers, The State University 